Publicación Semestral Pädi No. 10 (2018) 21-25

\title{
El lado químico de los cúmulos moleculares
}

\author{
Rojo Gómez Erik Gerardo ${ }^{\mathrm{a}}$, Tapia Benavides Antonio Rafael ${ }^{\mathrm{a}}$, Sánchez Cabrera Gloria ${ }^{\mathrm{a}}$, Zuno Cruz Francisco Javier ${ }^{\mathrm{a},{ }^{*}}$ \\ ${ }^{a}$ Universidad Autónoma del Estado de Hidalgo, Área académica de Química, Carr. Pachuca-Tulancingo Km 4.5, C. P. 42184, Mineral de la Reforma, Hidalgo, \\ México
}

\section{Resumen}

La química de los cúmulos metálicos ha sido estudiada a lo largo de los últimos años, debido al gran impacto que estos compuestos han tenido; principalmente por sus aplicaciones en diversas áreas de la ciencia y la tecnología. Aprovechando las propiedades y características con las que cuentan, los cúmulos metálicos han servido como catalizadores en diversos procesos químicos, aumentado el interés en el estudio de estos compuestos.

Palabras Clave: Cúmulo, metales de transición, ligantes, catálisis, química inorgánica.

\section{Introducción}

La palabra cúmulo, (del latín cumulus: montón, excedente) tiene significados diferentes dependiendo del contexto social, económico, tecnológico o científico en el que ésta se involucre. En este sentido, el ser humano ha relacionado el término cúmulo a los objetos (o fenómenos) más cotidianos que uno se pueda imaginar. Por ejemplo, los meteorólogos nombran "cúmulo" a la nube de base plana y forma voluminosa en la parte superior (que no produce lluvia). Por su parte, los astrónomos utilizan el término cúmulo para referirse a grandes agrupaciones de estrellas que se encuentran atraídas por efecto de su fuerza de gravedad. Un ejemplo de esto último son las congregaciones de estrellas que conforman a la vía láctea. Así, se podría seguir hablando de los diferentes significados que tiene la palabra cúmulo en biología, geología, sociología entre otros (Farlex, 2017) (figura 1).

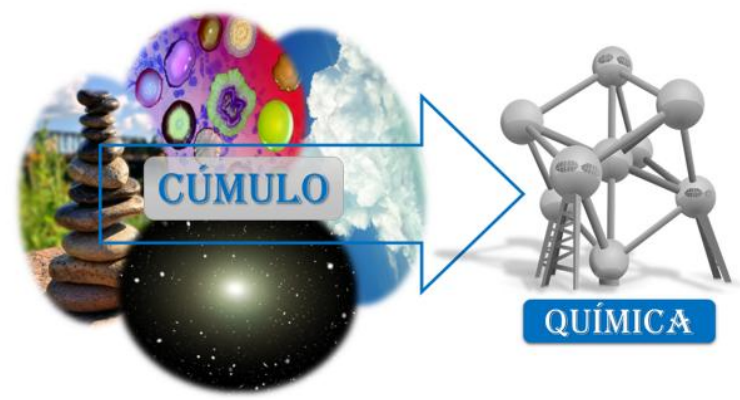

Figura 1: Definición de la palabra cúmulo.

Por supuesto que el concepto "cúmulo" no podía faltar en química. Si bien es sabido que dentro de las ciencias químicas existe una gran cantidad de compuestos de este tipo (tanto metálico como no metálico), este artículo se enfocará exclusivamente en aquellas especies cuya base estructural son átomos de metales de transición. Así, los cúmulos metálicos son compuestos que están formados por moléculas discretas en donde se tiene la presencia de varios átomos metálicos que pueden llegar a tener otros átomos o grupos de átomos unidos a ellos. Por lo tanto, en química un cúmulo puede ser definido como un agrupamiento o "racimo" de átomos o moléculas que quedan intermedias entre una estructura simple (molécula) y una estructura sólida (por ejemplo, una barra de oro puro) (Huheey, 1983) (figura 2).

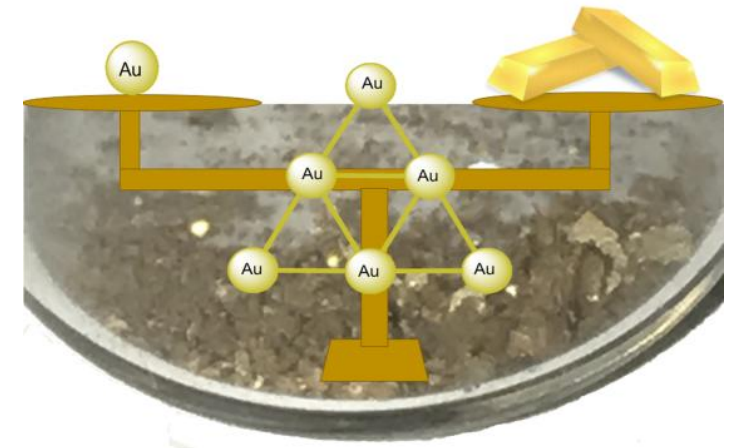

Figura 2: Definición de cúmulo en química.

Cabe resaltar que el interés por el estudio de este tipo de compuestos está dado por la relevancia química que tienen sus derivados. Además, la naturaleza de los enlaces metal-metal y las diferentes formas estructurales que estos compuestos pueden adoptar ha despertado la imaginación de no pocos de los mejores científicos en diferentes partes del mundo.

Es tanta la importancia que han adquirido estos compuestos que actualmente diversos grupos de investigación se encuentran desarrollando proyectos enfocados en la síntesis, caracterización, reactividad y aplicaciones potenciales de derivados de cúmulos metálicos. Ejemplo de esto último lo constituye el grupo de investigación de química organometálica del Área Académica de Química de la Universidad Autónoma del Estado de Hidalgo.

\section{Aspectos históricos}

\footnotetext{
* Autor en correspondencia. fjzuno@uaeh.edu.mx

Correos electrónicos: frik_gerardo@hotmai 1.com, tapiab@uaeh.edu.mx, gloriasa@uaeh.edu.mx

URL: https://sites.google.com/a/uaeh.edu.mx/uaeh_organometalica/home
} 
La química de metales de transición es un campo de estudio muy relevante dentro de la química inorgánica. Esto se debe a las aplicaciones múltiples que tienen los derivados metálicos, principalmente en la síntesis de materiales nuevos que puedan ser utilizados como catalizadores. Los procesos catalíticos industriales generalmente se llevan a cabo sobre una superficie metálica (catálisis heterogénea, figura 3). Para tratar de explicar las interacciones que se llevan a cabo a nivel microscópico se ha intentado usar como modelos de estos procesos químicos a compuestos mononucleares (es decir que contienen en su estructura un solo tipo de átomo metálico); sin embargo, en la actualidad se cree que compuestos con varios átomos en su estructura (cúmulos), puede representar de mejor forma estos procesos catalíticos heterogéneos.

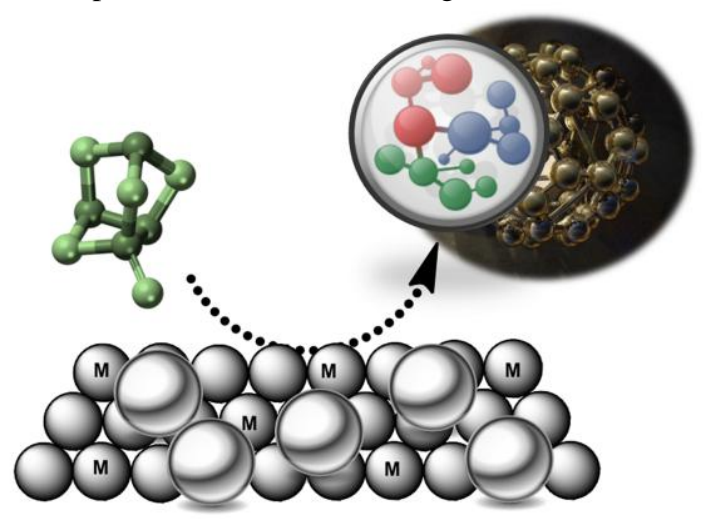

Figura 3: Proceso catalítico heterogéneo.

El conocimiento y la síntesis de compuestos en los cuales está involucrado un átomo metálico (complejos mononucleares) datan desde los albores de la química como ciencia. No obstante que el desarrollo sistemático de la química de compuestos con más de un centro metálico inició relativamente hace poco tiempo, se tiene reportado que los primeros compuestos bimetálicos sintéticos fueron los derivados de calomelanos, compuestos cuya estructura base está constituida por dos átomos de mercurio enlazados entre ellos y a su vez cada metal está enlazado a un átomo de cloro $\left(\mathrm{Hg}_{2} \mathrm{Cl}_{2}\right)$. Estos compuestos se utilizaban en la India alrededor del siglo XII como medicamentos y como base de cosméticos (cabe mencionar que los antiguos habitantes de la India no conocían la gran toxicidad de los calomelanos). No fue hasta comienzos del siglo XX que el interés por estos compuestos se enfocó en el estudio del enlace $\mathrm{Hg}-\mathrm{Hg}$ (Huheey, 1983).

A principios de la década de los sesentas comenzó a tener relevancia el estudio de la química de cúmulos metálicos, en esta época el químico estadounidense Frank Albert Cotton (profesor de la Universidad de Texas A\&M) definió a los cúmulos metálicos como especies químicas que contienen en su estructura tres o más átomos metálicos enlazados directamente entre ellos por la existencia de enlaces metalmetal (Dyson \& Mclndoe, 2000).

En la actualidad existe un gran número de cúmulo metálico que además contienen diversas moléculas o fragmentos orgánicos o inorgánicos (denominados "ligantes") enlazados a los centros metálicos. Dichos ligantes pueden ser por ejemplo, el grupo carbonilo $(\mathrm{CO})$, las fosfinas $\left(\mathrm{PR}_{3}\right)$, los tiolatos $\left(\mathrm{RS}^{-}\right)$ (en donde $\mathrm{R}=$ grupo alquilo, arilo o $\mathrm{H}$ ), el hidruro $\left(\mathrm{H}^{-}\right)$y los halogenuros $\left(\mathrm{F}^{-}, \mathrm{Cl}^{-}, \mathrm{Br}^{-}, \mathrm{I}\right)$ entre muchos otros (figura 4).
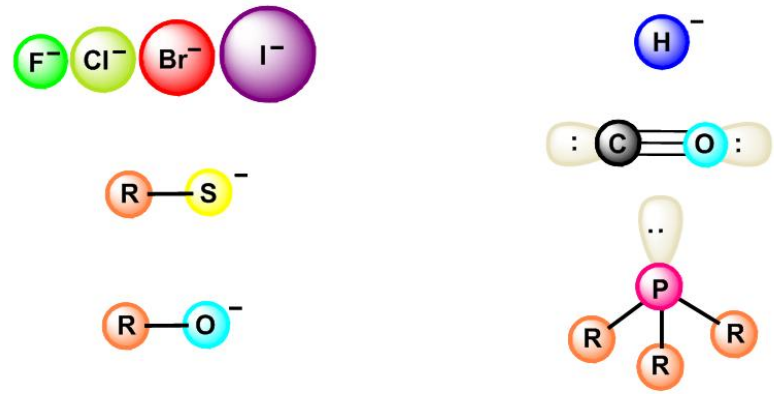

Ligantes donadores $\pi \quad \mathrm{R}=$ Arilo, alquilo Ligantes aceptores $\pi$

Figura 4: Ejemplos de moléculas orgánicas o inorgánicas denominadas "ligantes".

\section{Tipos de cúmulos.}

La gama de cúmulos metálicos es tal que es necesario realizar clasificaciones de estos compuestos. Por ejemplo, los científicos han efectuado ciertas clasificaciones en las cuales dichos compuestos se agrupan de acuerdo a las características de los componentes que conforman al cúmulo. La clasificación más utilizada está basada en la naturaleza de los ligantes enlazados al centro metálico. Por lo que, los cúmulos de metales de transición se pueden clasificar en tres grandes grupos (figura 5):

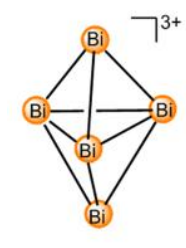

(a)

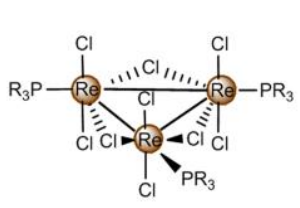

(b)

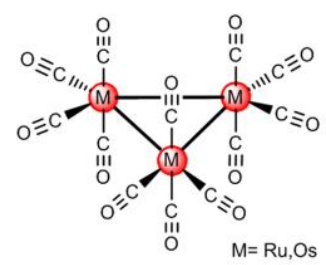

(c)
Figura 5: Ejemplos de cúmulos metálicos moleculares a) desnudo, b) con ligantes donadores $\pi$ y c) con ligantes aceptores $\pi$.

a) Cúmulos desnudos: Son especies metálicas que no contienen ligantes enlazados a su alrededor. Este tipo de cúmulos está más asociado a aquellos compuestos formados únicamente por metales del grupo principal ( $\mathrm{Ge}, \mathrm{Sn}, \mathrm{Pb}$ y $\mathrm{Bi}$ ) (Shiriver \& Kaez, 1990) (Mingos \& Wales, 1990) o a compuestos que de acuerdo al número de átomos de metales de transición que componen sus estructuras (de varias decenas a algunos cientos de átomos), se parecen más a las superficies metálicas continuas; a estos se les ha denominado nanopartículas metálicas por su tamaño.

b) Cúmulos con ligantes donadores $\pi$. Son compuestos que contienen átomos metálicos en estado de oxidación alto y a los cuales están unidos a ligantes de tipo donador $\pi$. Este tipo de ligantes se caracterizan por contener una alta densidad electrónica alrededor del átomo central, ejemplo de estos son los halogenuros, sulfuros, alcóxidos, entre otros (Shiriver \& Kaez, 1990) (Miessler, 1999).

c) Cúmulos con ligantes aceptores $\pi$. Son cúmulos que contienen átomos metálicos en estados de oxidación bajo. Dichos compuestos contienen en su estructura ligantes de tipo aceptor $\pi$. Cabe mencionar que los ligantes aceptores $\pi$ se caracterizan por su capacidad para aceptar densidad electrónica, el ejemplo principal de este tipo de ligantes lo 
constituye el CO (monóxido de carbono), el cual forma precursores químicos que permiten realizar la síntesis de cúmulos metálicos nuevos mediante su sustitución por otros ligantes como fosfinas, hidruros, etc. (Crabtree, 2005) (Cotton, 1999).

El balance entre la fuerza de los enlaces metal-ligante y metal-metal ha permitido sintetizar cúmulos desde tres hasta cincuenta átomos metálicos. Así, los cúmulos metálicos se encuentran en un punto intermedio entre los compuestos mononucleares y las nanopartículas. En la figura 6, se ilustra la progresión en el tamaño de partícula desde un compuesto con un centro metálico hasta superficies metálicas. (Dyson \& Mclndoe, 2000).
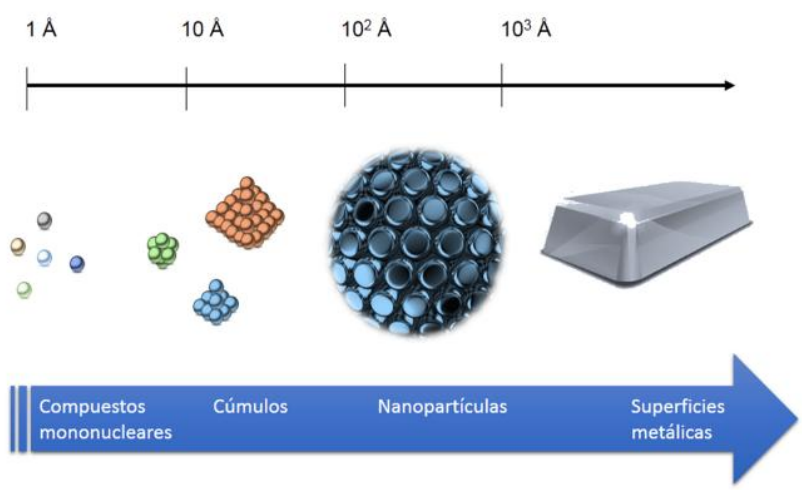

Figura 6: Tamaño relativo de cúmulos comparados con otros sistemas metálicos.

\section{Características de los cúmulos metálicos.}

Los cúmulos metálicos como se mencionó anteriormente, presentan características de acuerdo al tipo de ligante que tengan enlazado sobre los átomos metálicos, y serán estos ligantes los que definirán tanto su estabilidad como su reactividad. Por ejemplo, los cúmulos formados por elementos de la primera mitad del bloque de metales de transición (Sc, $\mathrm{Ti}, \mathrm{V}, \mathrm{Cr}, \mathrm{Y}, \mathrm{Zr}, \mathrm{Nb}, \mathrm{Mo}$ ) que tienen pocos electrones de valencia contienen principalmente ligantes donadores $\pi$ enlazados a los centros metálicos (los cuales donan densidad electrónica adicional al átomo metálico).

Debido a lo anterior, estos ligantes se pueden unir a dos o más átomos metálicos a la vez formando puentes. Este tipo de coordinación se simboliza con la letra griega " $\mu$ " que indica que un ligante en particular está enlazado a dos o más átomos metálicos del compuesto y la cantidad de átomos que participan en la formación del puente se representa con un subíndice numérico. Cabe añadir que "los puentes" se pueden formar a lo largo de un borde o de una cara del cúmulo metálico (tabla 1).

Tabla 1. Características electrodonantes de ligantes comunes coordinados de forma puente en cúmulos metálicos.

\begin{tabular}{|c|l|}
\hline Electrones donados & \multicolumn{1}{c|}{ Ligantes } \\
\hline $\begin{array}{c}\text { Puente a un enlace } \mathrm{M}-\mathrm{M}\left(\mu_{2}\right) \\
\bullet \quad \text { Donador de dos electrones }\end{array}$ & $\mathrm{O}, \mathrm{S}, \mathrm{Se}$ \\
\hline$\bullet \quad$ Donador de tres electrones & $\mathrm{SR}, \mathrm{OR}, \mathrm{PR}_{2}, \mathrm{AsR}_{2}, \mathrm{Cl}, \mathrm{Br}$ \\
\hline Puente a una cara trinuclear $\mathrm{M}_{3}\left(\mu_{3}\right)$ & \\
\hline$\bullet \quad$ Donador de dos electrones & $\mathrm{O}, \mathrm{S}, \mathrm{Se}, \mathrm{PR}, \mathrm{AsR}$ \\
\hline$\bullet \quad$ Donador de tres electrones & $\mathrm{Cl}, \mathrm{I}, \mathrm{Br}$ \\
\hline Puente a una cara tetranuclear $\mathrm{M}_{4}\left(\mu_{4}\right)$ & \\
\hline$\bullet \quad$ Donador de tres electrones & $\mathrm{PR}, \mathrm{S}$ \\
\hline \hline $\mathrm{M}=$ metal; $\mathrm{R}=$ alquilo o arilo &
\end{tabular}

Por otro lado, los cúmulos formados por elementos de la segunda mitad del bloque "d" (Mn, Fe, Co, Ni, Cu, Ru, Rh, $\mathrm{Pd}, \mathrm{Os}, \mathrm{Au}$ ), que contienen muchos electrones de valencia, se enlazan preferentemente a ligantes tipo aceptor $\pi$. Estos ligantes son capaces de aceptar densidad electrónica del cúmulo metálico reforzando el enlace metal-ligante, lo que ayuda a estabilizar a toda la molécula (Shiriver \& Kaez, 1990).

Además de donar o retirar densidad electrónica al cúmulo metálico, los ligantes juegan otro papel importante dentro de la estabilidad del cúmulo. En conjunto con el número de átomos metálicos presentes en el compuesto, los ligantes influyen y cambian el arreglo espacial de la molécula y por lo tanto éstos sustituyentes afectan la geometría que adoptará el fragmento metálico del compuesto. Las formas geométricas que adopta un cúmulo son variadas y esto permitirá que la especie sea más estable o que la reactividad que presente sea diferente; a continuación, se hará una descripción general del arreglo espacial que los cúmulos pueden presentar con base en el número de átomos metálicos que lo conforman (Shiriver \& Kaez, 1990).

a) Cúmulos con tres átomos metálicos: El principal arreglo que presenta un cúmulo con tres átomos metálicos en su estructura (de acuerdo al principio de que los tres metales deben de estar enlazados directamente entre ellos) es la de forma triangular (figura 7a). En estos sistemas las distancias de enlace M-M se encuentran en un rango entre 2.90 y $3.15 \AA$ (dependiendo del metal) y en conjunto contienen 48 electrones de valencia. Se han reportado diversos ejemplos de estos cúmulos que contienen en su estructura como ligante principal al grupo carbonilo (CO), pero también pueden incluir ligantes como alquinos, tiolatos o fosfinas. En la figura $7 \mathrm{~b}$ se muestra la estructura del compuesto de rutenio $\left[\mathrm{Ru}_{3}(\mathrm{CO})_{9}(\mu-\mathrm{H})\left\{\mu_{3^{-}}\right.\right.$ $\left.\mathrm{C} \equiv \mathrm{C}\left(\mathrm{C}_{6} \mathrm{H}_{2}-2,4,5-\left(\mathrm{CH}_{3}\right)_{3}\right\}\right]$ (Hernandez-Sandoval, et al., 2011) y en la figura $7 \mathrm{c}$ se muestra la estructura del compuesto tipo estrella $\left[\left\{\mathrm{Os}_{3}(\mu-\mathrm{H})(\mathrm{CO})_{10}\right\}_{3}\left\{1,3,5-\left(\mu-\mathrm{SCH}_{2}\right)_{3} \mathrm{C}_{6} \mathrm{H}_{3}\right\}\right]$, formado por tres cúmulos trinucleares de osmio unidos a través de un ligante tri-tiolato (Sánchez-Cabrera, et al., 2014).

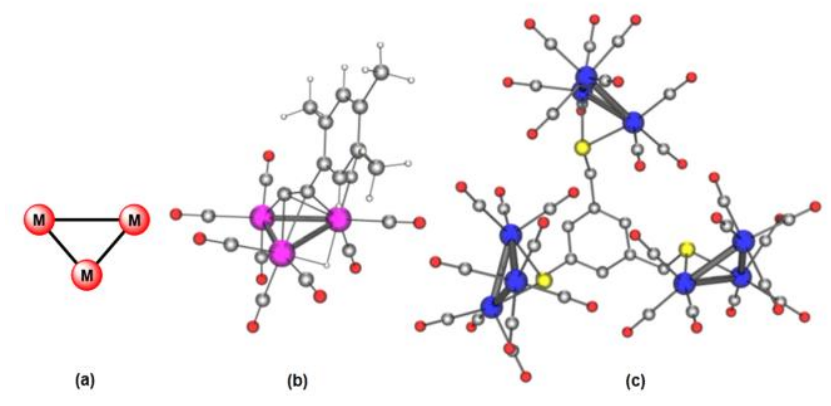

Figura 7: a) Arreglo geométrico de cúmulos trinucleares. b) y c) ejemplos de cúmulo trinucleares.

b) Cúmulos con cuatro átomos metálicos: Los cúmulos con cuatro átomos metálicos pueden presentar más de un arreglo geométrico, que depende del número de electrones de valencia que contenga el compuesto y del número y tipo de ligantes que contenga enlazados (factores estéricos); los arreglos más comunes son: la forma de tetraedro (60 electrones de valencia), la de mariposa y la cuadrada plana (ambas con 64 electrones de valencia) (figura 8a). En la figura $8 \mathrm{~b}$ se muestra un ejemplo de un cúmulo tetranuclear de rutenio con ligantes carbonilos y enino $\left[\mathrm{Ru}_{4}(\mu-\mathrm{CO})(\mathrm{CO})_{10}\left(\mu_{4}-\mathrm{C}_{5} \mathrm{H}_{6}\right)_{2}\right]$ con geometría de mariposa (Zuno-Cruz, et al., 2002). En la 
figura $8 \mathrm{c}$ se muestra un ejemplo de un cúmulo tetranuclear de rutenio con ligantes carbonilos, bencino, fosfina y fosfinideno $\left[\mathrm{Ru}_{4}(\mathrm{CO})_{9}(\mu-\mathrm{CO})\left\{\mu_{4}-\mathrm{PCH}_{2} \mathrm{CH}_{2} \mathrm{P}\left(\mathrm{C}_{6} \mathrm{H}_{5}\right)_{2}\right\}\left(\mu_{4}-\mathrm{C}_{6} \mathrm{H}_{4}\right)\right]$, con geometría cuadrada (Sánchez-Cabrera, et al., 2002).

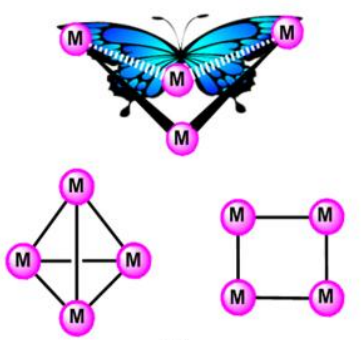

(a)

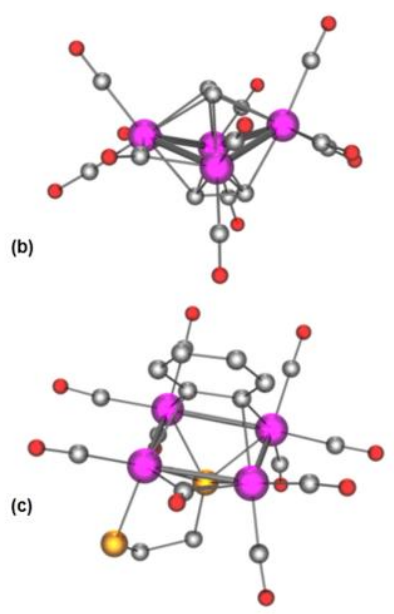

Figura 8: a) Arreglo espacial de cúmulos tetranucleares. b) y c) ejemplos de cúmulos tetranucleares (los átomos de hidrógeno y algunos átomos de carbono se han omitido por claridad).

c) Cúmulos con cinco y seis átomos metálicos: El arreglo espacial para cúmulos con cinco átomos metálicos va desde una forma de bipirámide trigonal, pirámide de base cuadrada, tetraedro apicado, hasta una forma de corbatín, entre otros. En cuanto a los cúmulos con seis átomos metálicos se encuentra principalmente la forma de octaedro, prisma triangular o bi-tetraédrica. Es importante mencionar que existen cúmulos con un número superior a los siete átomos metálicos y por consecuencia su conteo electrónico y variedad estructural será superior a los antes mencionados (figura 9).
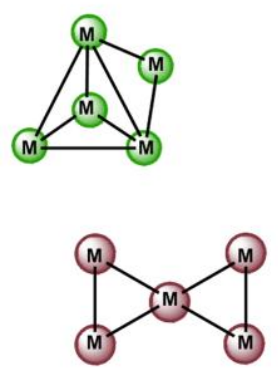
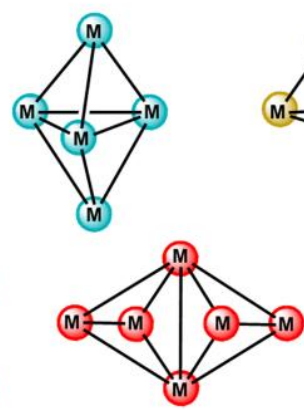
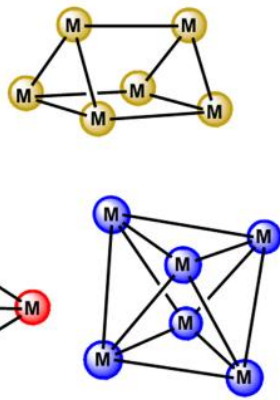

Figura 9: Arreglo estructural de cúmulos penta- y hexanucleares.

En cuanto a la reactividad de este tipo de compuestos, se sabe que pueden ocurrir diversos procesos químicos sobre sus caras o bordes, ya sean reacciones de sustitución, reacciones óxido-reductivas, reacciones fotolíticas o diversos procesos catalíticos tales como hidrogenaciones, isomerizaciones $\mathrm{u}$ oligomerizaciones para la obtención de compuestos orgánicos.

\section{Aplicaciones.}

Como se comentó, alrededor de la estructura metálica del cúmulo es posible que se lleven a cabo diversas reacciones químicas; sin embargo, el estudio en cuanto a las aplicaciones de estos compuestos es un tema que permite tener grandes oportunidades para desarrollar investigación científica y tecnológica de alto nivel (figura 10).

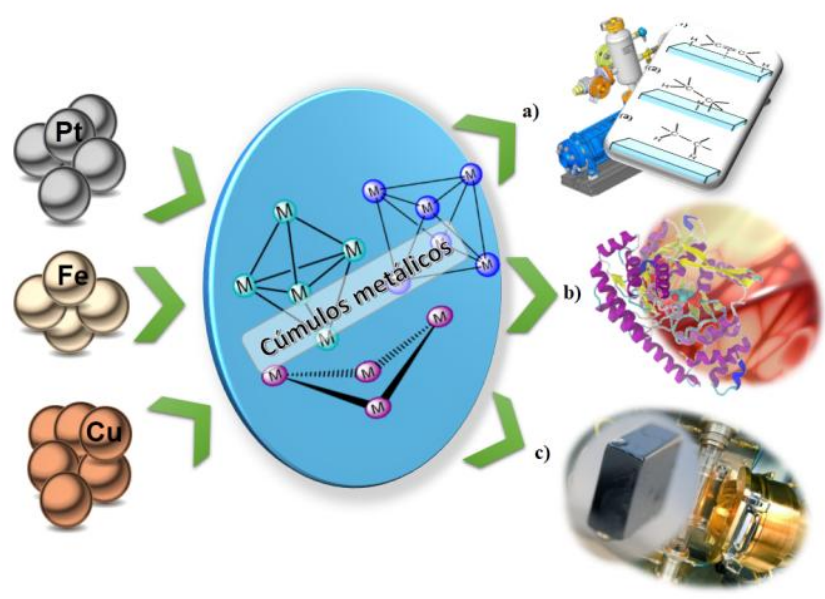

Figura 10: Aplicaciones de los cúmulos metálicos. a) Catálisis y síntesis de nuevos compuestos. b) Aplicaciones biológicas y biomédicas. c) Síntesis de materiales de alta tecnología.

La principal área de aplicación de estos compuestos recae en el desarrollo de catalizadores (en el que la base estructural es un cúmulo ya sea con o sin ligantes coordinados). Los catalizadores son compuestos que aceleran una reacción química sin participar en ella, principalmente para la obtención de compuestos orgánicos (reacciones de oligomerización, hidrogenación de olefinas, etc.) o para la degradación a moléculas más simples de compuestos orgánicos azufrados (Girgis, 1991) o nitrogenados (que pueden ser potencialmente dañinos para la salud del ser humano y la del medio ambiente). Un ejemplo de esto es el proceso de hidrodesulfuración en donde se remueve el azufre que contienen los derivados del petróleo, cuya combustión producirá óxidos de azufre que son algunos de los responsables de la contaminación ambiental y de la formación de la llamada "lluvia ácida" (Braustein, 1999).

En la industria petroquímica se han usado cúmulos de platino debido a que estos compuestos se utilizan como catalizadores en el proceso de platforming del petróleo. En tanto, cúmulos de hierro se han utilizado en la síntesis de compuestos orgánicos en la formación de enlaces C-C, utilizando los centros metálicos como análogo a la superficie de un catalizador heterogéneo, en donde se lleva a cabo el procesos de transformación de los fragmentos orgánicos (Yamamoto, 1986).

En lo referente a las aplicaciones biológicas de estos compuestos se ha reportado que los derivados de cúmulos de hierro del tipo $\left[\mathrm{Fe}_{4} \mathrm{~S}_{4}(\mathrm{SR})_{4}\right]^{\mathrm{n}-}$ son de importancia científica para comprender el papel que juega el fragmento hierro-azufre en el comportamiento químico de proteínas, así como de los procesos redox que pueden ocurrir en estas biomoléculas (Shiriver \& Kaez, 1990). Adicionalmente, existen nanocúmulos de $\mathrm{Ag}$ y $\mathrm{Au}$ con propiedades fluorescentes que permiten su aplicación como marcadores en procesos biológicos (Shang \& Dong, 2011).

Otra de las áreas de aplicación de los cúmulos metálicos la constituye el desarrollo de materiales nuevos, en donde se han podido sintetizar compuestos con varias decenas de átomos metálicos generalmente sin ligantes, conocidos como nanopartículas, en ellos se ha propuesto que su superficie metálica maximizaría los procesos catalíticos. Asimismo, se ha buscado aprovechar la estabilidad y propiedades de algunos 
cúmulos de $\mathrm{Cu}$ (Badri \& Pathak, 2013), o de Au con alta nuclearidad $\left(\mathrm{Au}_{309}\right)$, que permitan tener propiedades de superconducción (Wuelfin \& Green, 2000) y que podrían ser muy útiles para el desarrollo tecnológico actual.

\section{Conclusiones.}

Como se ha descrito, los cúmulos metálicos son especies químicas únicas cuyas propiedades químicas permiten que su área de desarrollo y aplicación sea muy grande. Sin duda alguna, aún queda mucho que aprender acerca de estos compuestos y es necesario seguir investigando esta área de las ciencias que permita entender su comportamiento químico básico. Para de esta forma tener la capacidad de explotar al máximo las propiedades que permitan desarrollar nuevos materiales y diseñar tecnologías novedosas para combatir de manera eficaz las problemáticas ambientales o de energía entre muchas otras, que hoy en día preocupan no solo a nuestro país, sino al mundo entero.

\section{English Summary}

\section{THE CHEMICAL SIDE OF MOLECULAR CLUSTERS}

\section{Abstract}

The chemistry of metal clusters has been studied over the past years because these compounds have great applications in many areas of science and technology. Taking advantage of their chemical properties, metal clusters have been used as catalysts in some chemical processes, increasing the interest in their study.

Keywords:

Cluster, transition metals, ligands, catalysis, inorganic chemistry

\section{Agradecimientos}

EGRG agradece a CONACYT por la beca otorgada para sus estudios de maestría.

A CONACYT por los proyectos de Ciencias Básicas CB2011/169067 y de cátedras 2016 no. 222.

A la Universidad Autónoma del Estado de Hidalgo por los proyectos PAI-UAEH-2015-3313 y PAI-UAEH-2015-3289.
Al proyecto de Red Temática de Colaboración Académica (PRODEP-2015)

\section{Referencias}

Badri, Z., \& Pathak, S. (2013). All-Metal Aromaticity: Revisiting the Ring Current Model Among. J. Chem. Theory Comput., 9 (11) 4789-4796.

Braustein, P. (1999). Metal Clusters in Chemistry. Alemania: Wiley$\mathrm{VCH}$.

Cotton, F. A. (1999). Química Inorgánica Avanzada. México: Limusa Crabtree, R. (2005 ). The Organometallics Chemistry of the Transitions Metals. New Jersey, EUA: John Wiley \& Sons. Dyson, \& Mclndoe. (2000). Transtition Metal Carbonyl Clusters Chemistry. Inglaterra: University of Cambridge.

Farlex. (16 de Mayo de 2017). The Free Dictionary. Obtenido de The Free Dictionary: http://es.thefreedictionary.com

Girgis, M. J. (1991). Reactivities, reaction networks, and kinetics in high-pressure catalytic hydroprocessing. Ind. Eng. Chem. Res, 9 (30), 2021-2058.

Hernandez-Sandoval, M., Sánchez-Cabrera, G., Rosales-Hoz, M. J., Leyva, M. A., Salazar, V., Alvarado-Rodriguez, J. G., \& Zuno-Cruz, F. J. (2011). C-H and C-C bond activations of terminal alkynes in the presence of a butterfly-shaped heteronuclear $\mathrm{Ru}_{3} \mathrm{Au}$ cluster. J. Organomet. Chem, 696 (25), 4070-4078.

Huheey, J. (1983). Inorganic Chemistry. New York, EUA: Harper and Row.

Miessler, G. L. (1999). Inorganic Chemistry. Upper Saddle River, NJ, EUA: Tarr. Prentice-Hall.

Mingos, M. P., \& Wales. (1990). Introduction to Cluster Chemestry. New York, EUA: Prentice-Hall Inc. Publisher.

Sánchez-Cabrera, G., Zuno-Cruz, F. J., López-Ruiz, H., Rosales-Hoz, M. J., Leyva, M. A., \& Rodríguez, J. A. (2014). The synthesis and structural characterization of $\mathrm{C}_{3}$ symmetry "star-like" cluster $\left[\mathrm{fOs}_{3}(\mu-\right.$ $\left.\left.\mathrm{H})(\mathrm{CO})_{10}\right\}_{3}\left\{1,3,5-\left(\mu-\mathrm{SCH}_{2}\right)_{3} \mathrm{C}_{6} \mathrm{H}_{3}\right\}\right]$. J. Organomet. Chem., 751, 826-829. Sánchez-Cabrera, G., Zuno-Cruz, F. J., Rosales-Hoz, M. J., \&

Bakhmutov, V. I. (2002). Pyrolysis of $\left[\mathrm{Ru}_{4}(\mathrm{CO})_{10}(\mathrm{dppe})\right]$ : Activation of C$\mathrm{H}$ and $\mathrm{P}-\mathrm{Ph}$ Bonds. The Crystal Structure and Dynamical Behavior of $\left[\mathrm{Ru}_{4}(\mathrm{CO})_{9}(\mu-\mathrm{CO})\left\{\mu_{4}-\eta^{2}-\mathrm{PCH}_{2} \mathrm{CH}_{2} \mathrm{P}\left(\mathrm{C}_{6} \mathrm{H}_{5}\right)_{2}\right\}\left(\mu_{4}-\eta^{4}-\mathrm{C}_{6} \mathrm{H}_{4}\right)\right] . J$. Organomet. Chem., 660 (2), 153-160.

Shang, L., \& Dong, S. (2011). Ultra-Small Fluorescent Metal Nanoclusters: Synthesis and Biological Applications. Nanotoday, 6, 401418 .

Shiriver, D., \& Kaez. (1990). The Chemistry of Metal Cluster Complexes. New York, EUA: VCH Publisher.

Wuelfin, P., \& Green, S. (2000). Electronic Conductivity of SolidState, Mixed-Valent, Monolayer-Protected Au Clusters. J. Am. Chem Soc., 122 (46), 11465-11472.

Yamamoto, A. (1986). Organotransition Metal Chemistry: Fundamental Concepts and Application. New York, EUA: WileyInterscience.

Zuno-Cruz, F. J., Sánchez-Cabrera, G., Rosales-Hoz, M. J., \& Nöth, H. (2002). The Reaction of $\left[\mathrm{H}_{4} \mathrm{Ru}_{4}(\mathrm{CO})_{12}\right]$ with 1-penten-3-yne: Dimerization and Trimerization Through the Triple Bonds. J. Organomet. Chem., 649 (1), 43-49. 